\title{
MODELS OF TERAHERTZ AND INFRARED DEVICES BASED ON GRAPHENE/ BLACK-ASP HETEROSTRUCTURES
}

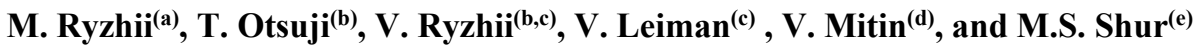 \\ (a)University of Aizu, Aizu-Wakamatsu, Japan \\ (b) RIEC, Tohoku University, Sendai, Japan \\ (c) Moscow Institute of Physics and Technology, Dolgoprudny, Russia \\ (d)University at Buffalo, Buffalo, USA

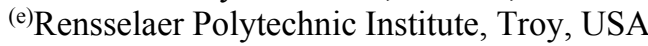 \\ (a)m-ryzhii@u-aizu.ac.jp
}

\begin{abstract}
The gapless energy spectrum of the graphene layers (GLs) enables the interband absorption and emission of photons and plasmons in the terahertz (THz) and infrared (IR) spectral range. The energy of the emerging the black-phosphorus (b-P), black-arsenic (b-As), and the compounds $\left(\mathrm{b}-\mathrm{A} \mathrm{s}_{\mathrm{x}} \mathrm{P}_{1-\mathrm{x}}\right)$ varies from 0.15 to $1.7 \mathrm{eV}$, depending on the number of the atomic sheets and the component relative content. Due to a strong anisotropy of the b-P and b-As, the ratios of the carrier effective masses in different in-plain directions are very large. One of the crucial properties of the GL heterostructures with the b-P, b-As, and b- $\mathrm{As}_{\mathrm{x}} \mathrm{P}_{1-\mathrm{x}}$ barrier layers are associated with the GL Dirac point corresponding to the energy gap in the barriers. Combination of GLs with the $\mathrm{b}-\mathrm{P}, \mathrm{b}-\mathrm{As}$, and $\mathrm{b}-\mathrm{As}_{\mathrm{x}} \mathrm{P}_{1-\mathrm{x}}$ layers opens new prospects for the novel $\mathrm{THz}$ and IR devices, in particular, GL-based photodetectors, electro-optical modulators, and sources of $\mathrm{THz} / \mathrm{IR}$ radiation, including the lasers with the GL active region.
\end{abstract}

Keywords: black-phosphorus, black-arsenic, graphene, heterostructure, terahertz, infrared

\section{INTRODUCTION}

In this work, we demonstrate the concepts and models of $\mathrm{THz}$ and IR photodetectors, switches, sources, and modulators based on the heterostructures based on the graphene layers (GLs) (Castro Neto 2009) and the black-P (b-P), black-As (b-As), and black- $\mathrm{As}_{\mathrm{x}} \mathrm{P}_{1-\mathrm{x}}$ (b$\mathrm{As}_{\mathrm{x}} \mathrm{P}_{1-\mathrm{x}}$ ) layers (Keyse 1953, Morita 1986, Asashina 1984, Ling 2015, Xia 2014, Guo 2015, Liu 2015, Long 2017, Yuan 2018). Our consideration is based on our recent results (Ryzhii 2017a, Ryzhii 2017b, Ryzhii 2017c, Ryzhii 2018a, Ryzhii 2018b, Ryzhii 2019a, Ryzhii 2019b, Ryzhii 2019c).

In this presentation, we mainly focus on prospective $\mathrm{THz} / \mathrm{IR}$ sources (including lasers) using the interband transitions in the optically pumped GLs.

We consider also the THz/IR sources based on GL active region with the lateral electron injection from the side contacts and the hole vertical injection via the b-P (b-As) layer.
The optical pumping can be realized using mid- or nearIR light-emitting diodes (LEDs), in particular, integrated with the laser-active GL structure. The main problem is that the electron-hole pairs generated by such LEDs in the GL have fairly large energies. This results in relatively high effective temperature of the electron-hole plasma in the GL that hampers the achievement of the interband population inversion and the possibility of lasing. This obstacle can be avoided introducing the absorbing-cooling layer with the sufficiently narrow energy gap.

The sketch of the band diagram of the GL-based laser structure with the absorbing-cooling layer integrated with the pumping LED is shown in Fig. 1.

The b-As or b-As $\mathrm{s}_{\mathrm{x}} \mathrm{P}_{1-\mathrm{x}}$ absorbing-cooling layers can be particularly effective because their energy gap $\Delta_{\mathrm{G}}$ rather small (up to $0.15 \mathrm{eV}$ ).

This implies that the energy of the electron-hole pairs injected into GL can be about the latter value even in the case of optical pumping with considerably highenergy photons. As shown, if the energy of the injected pairs is smaller than the energy of optical phonons in GLs (about $0.2 \mathrm{eV}$ ), the effective temperature of the electron-hole plasma in the laser-active GL can be lower than the ambient (lattice) temperature. This is beneficial for the population inversion in the GL.

\section{MODELS AND RESULTS}

\subsection{Optical pumping via b-AsP absorbing-cooling layer}

We developed the model for the THz and FIR lasers with the band diagram of Fig. 1. The device comprises the $b-A s_{x} P_{1-x}-G L$ heterostructure playing the role of the $\mathrm{THz}$ active region mounted on the top of the $\mathrm{P}^{+}-\mathrm{i}-\mathrm{N}^{+}$ LED heterostructure. The active region and LED are separated by a wide-gap transparent barrier layer. The IR radiation (with the photon energy $\hbar \Omega$ ) generated by the LED passes the barrier layer and produces the fairly hot electrons and holes in the $\mathrm{b}-\mathrm{As}_{\mathrm{x}} \mathrm{P}_{1-\mathrm{x}}$ absorbingcooling layer. If the thickness of the $b-\mathrm{As}_{\mathrm{x}} \mathrm{P}_{1-\mathrm{x}}$ layer substantially exceeds the characteristic cooling length, 


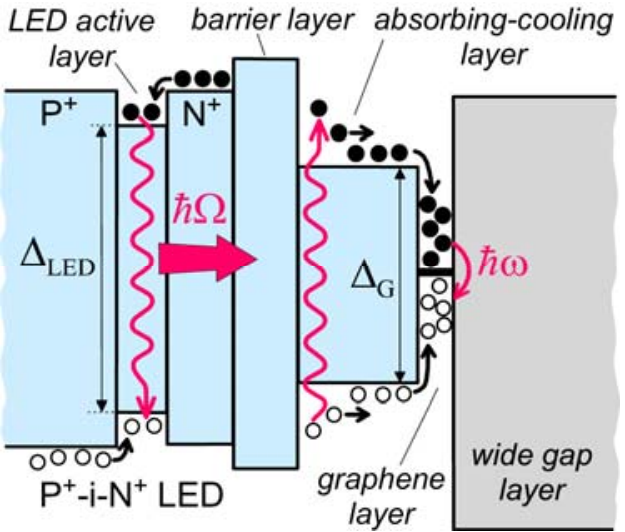

Figure 1: Band diagram of the GL-based laser with an absorbing-cooling layer integrated with a pumping LED.
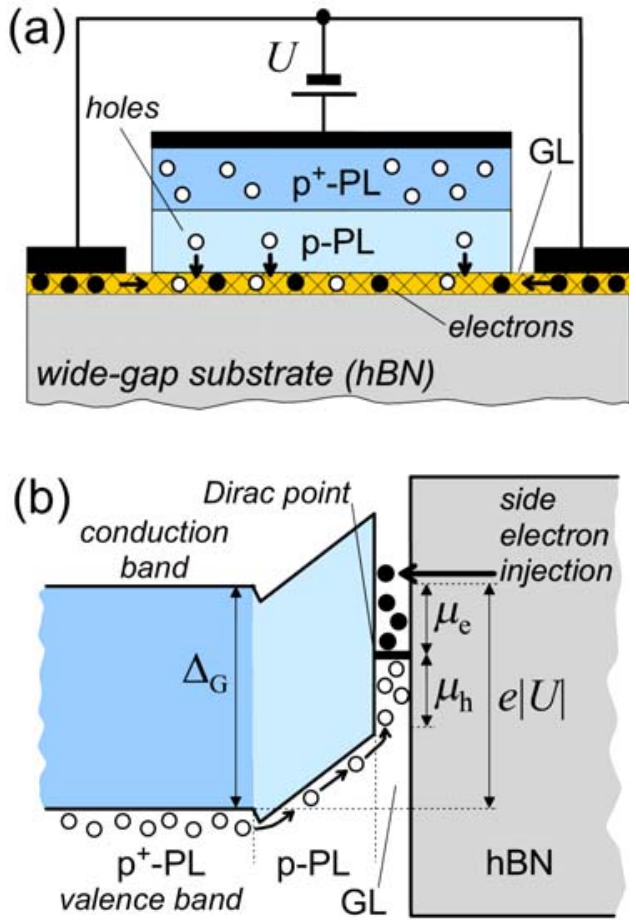

Figure 2: Sketch of the heterostructure with b-P layer (PL) and with lateral electron and vertical hole injection (a), and its energy band diagram (b).

the photogenerated carriers arrive at the GL being efficiently cooled down. The injection into the GL of the carriers with the energy smaller than the energy of optical phonons in the GL can result in reinforcement of the interband population inversion in the GL.

Using the model, we calculated the effective temperature $T$ of the electron-hole plasma and its quasiFermi energy $\mu$ at different pumping conditions. It is assumed the absorbing-cooling layers constitute the $\mathrm{b}$-As and b-P layers consisting of sufficiently large number of the atomic sheets $(>8)$, so that the energy gaps $\Delta_{\mathrm{G}}$ were set to be 0.15 and $0.3 \mathrm{eV}$, respectively.

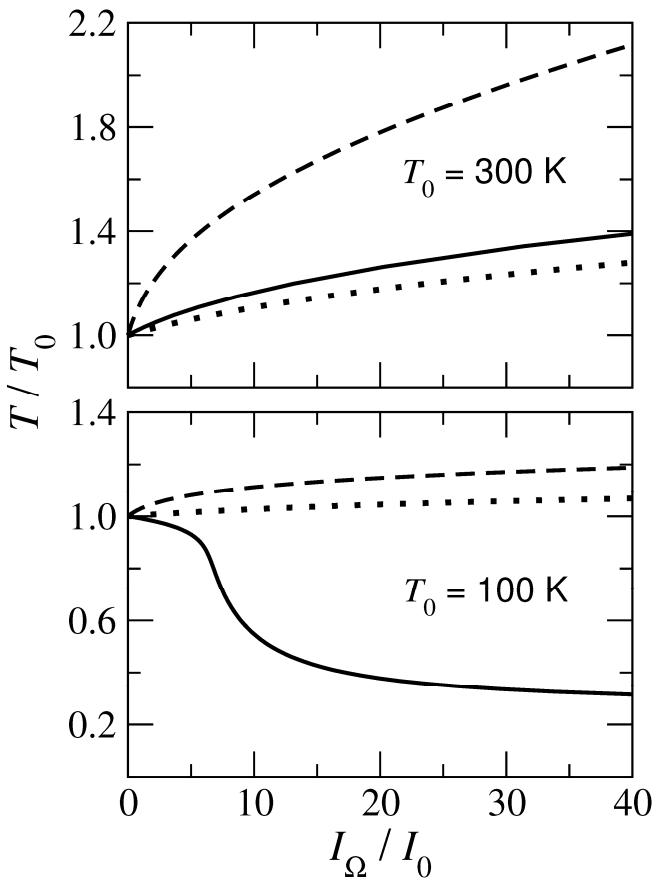

Figure 3: The effective temperature of the electron-hole plasma in the GL normalized by the lattice temperature $T_{0}$ as function of the normalized intensity of the pumping radiation $I / I_{0}$ calculated for different $T_{0}$ for b-As (solid lines) and b-P (dashed lines) absorbingcooling layers at energy of pumping photons $\hbar \Omega=0.36 \mathrm{eV}$. Dotted lines correspond to the direct pumping (without the absorbing-cooling layer).

Figure 2 shows the heterostructures with relatively narrow-gap p-type b-P (or b-As) layer on a wide-gap substrate and its energy band diagram at the operating voltage $U$. Different relatively wide-gap materials can be utilized, for example, hexagonal Boron Nitride (hBN).

A wide gap in the hBN substrate provides high energy barrier for the electrons and holes in the GL and blocks their leakage to the substrate. At the applied bias voltage the electrons can freely occupy the GL. Simultaneously, the holes pass vertically from the heavily-doped $\mathrm{p}^{+}$region through the undoped or lightly doped b-P layer are vertically injected into the GL. Due to the finite energy spacing between the valence band of the hole injector and the Dirac point in the GL, the injected holes can bring a marked energy into the electron-hole system in the GL. However, the injected energy is effectively removed due to the emission of the high-energy (about $200 \mathrm{meV}$ ) optical phonons in the GL. This can result in the cooling of the carrier system injected into the GL.

Figures 3 and 4 demonstrate examples of the calculated dependences of effective temperature of the electronhole plasma in the GL normalized by the lattice temperature $T_{0}$ and the quasi-Fermi energy $\mu$ as functions of the normalized intensity of the pumping radiation $I / I_{0}$ for the devices with $b$-As and $b-P$ layers. 


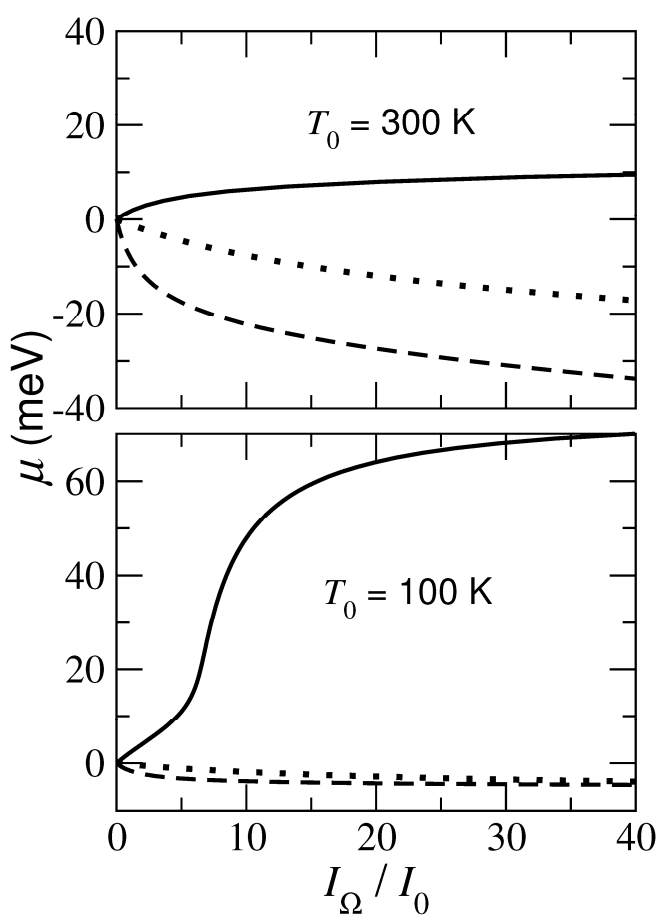

Figure 4: The quasi-Fermi energy as function of the normalized intensity of the pumping radiation $I / I_{0}$ calculated for different $T_{0}$ for b-As (solid lines) and b-P (dashed lines) absorbing-cooling layers at energy of pumping photons $\hbar \Omega=0.36 \mathrm{eV}$. Dotted lines correspond to the direct pumping (without the absorbing-cooling layer).

In particular, one can see that in the case b-As absorbing-cooling layer at lowered lattice temperatures (see bottom panel of Fig. 3 for $T_{0}=100 \mathrm{~K}$ ), the pumping can lead to a marked cooling down of the electron-hole plasma in the GLs. However, in the devices with the b-P absorbing-cooling layer or without the latter (direct optical pumping) $\mu<0$, i.e., the population inversion is not achieved, at least at the given energies of the pumping photons (Fig. 4).

\subsection{Vertical hole pumping via $b$-As layer}

Figure 5 shows the sum of the electron and hole quasiFermi energies $\left(\mu_{\mathrm{e}}+\mu_{\mathrm{h}}\right)$, the effective temperature $T$ of the system, and normalized net quasi-Fermi energy $\left(\mu_{\mathrm{e}}+\mu_{\mathrm{h}}\right) / T$ calculated as functions of the normalized injection current $j / j_{\mathrm{G}}$ for different relative strengths, $s$, of the carrier interaction with the GL and substrate surface optical phonons. Small values of $s$ correspond to relatively weak interaction with the substrate surface optical phonons. The energy of these phonons is assumed to be $100 \mathrm{meV}$. The lattice temperature and the characteristic current density are set $T_{0}=25 \mathrm{meV}$ and $j_{\mathrm{G}}=160 \mathrm{~A} / \mathrm{cm}^{2}$, respectively. As seen from Fig. 5, the pumping method under consideration can result in fairly high values of the carrier Fermi energy.
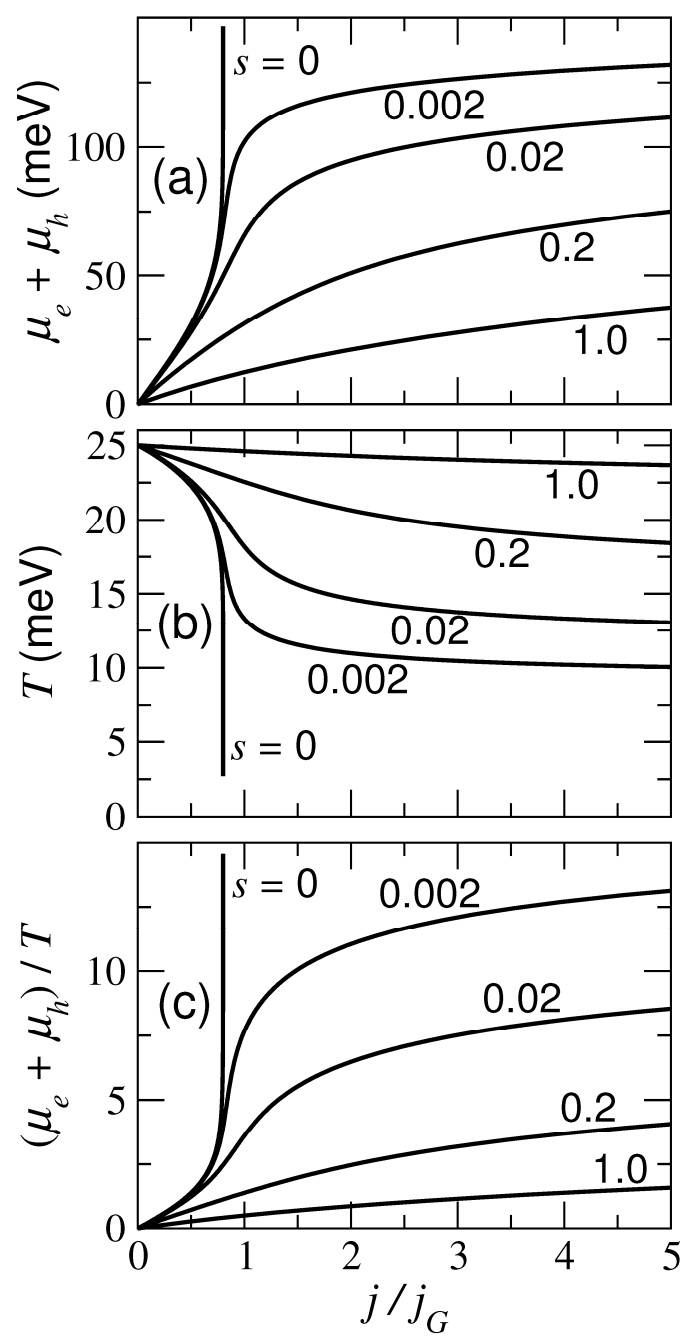

Figure 5: Dependences of (a) the net quasi-Fermi energy $\left(\mu_{\mathrm{e}}+\mu_{\mathrm{h}}\right)$, (b) carrier effective temperature $T$, and (c) normalized net quasi-Fermi energy $\left(\mu_{\mathrm{e}}+\mu_{\mathrm{h}}\right) / T$ as functions of the normalized injection current $j / j_{\mathrm{G}}$ for different relative strengths $s=0-1.0$.

Decrease in the carrier effective temperature $T$ below the lattice temperature $T_{0}$, and, therefore, a strong degeneration of the carrier system in the GL are beneficial for the interband THz/IR lasing.

\section{CONCLUSIONS}

We demonstrated that the combination of GLs with the b-As and b-P layers exhibits a great potential for novel $\mathrm{THz}$ and IR devices. In particular, the GL/b-AsP heterostructures are promising for the $\mathrm{THz}$ and IR lasers using the interband transitions in the active GLs.

\section{ACKNOWLEDGMENTS}

The work was supported by the Japan Society for Promotion of Science, KAKENHI Grant No. 16H06361, RIEC Nation-Wide Collaborative Research Project, and by the Office of Naval Research (Project Monitor Dr. Paul Maki). 


\section{REFERENCES}

Castro Neto A.H., Guinea F., N. Peres M.R., Novoselov K.S., Geim A.K., 2009. The electronic properties of graphene. Review of Modern Physics, 81, 109162.

Keyes R.W., 1953. The electrical properties of black phosphorus. Physical Review, 92 (3), 580-584.

Morita A., 1986. Semiconducting black phosphorus. Applied Physics A, 39(4), 227-242.

Asahina H., Morita A., 1984. Band structure and optical properties of black phosphorus. Journal of Physics C: Solid State Physics, 17(11), 1839-1852.

Ling Xi, Wang H., Huang S., Xia F., Dresselhaus M.S., 2015. The renaissance of black phosphorus. Proceedings of the National Academy of Sciences of the USA, 112(15), 4523-4530.

Xia F., Wang H., Jia Y., 2014. Rediscovering black phosphorus as an anisotropic layered material for optoelectronics and electronics. Nature Communications, 5, 4458.

Guo Z., Zhang H., Lu S., Wang Z., Tang S., Shao J., Sun Z., Xie H., Wang H., Yu X.-F., Chu P.K., 2015. From black phosphorus to phosphorene: basic solvent exfoliation, evolution of Raman scattering, and applications to ultrafast photonics. Advanced Functional Materials, 25(45), 69967002.

Liu B., Kopf M., Abbas A.N., Wang X., Guo Q., Jia Y., Xia F., Weihrich R., Bachhuber F., Pielnhofer F., Wang H., Dhall R., Cronin S.B., Ge M., Fang X., Nilges T., and Zhou C., 2015. Black arsenicphosphorus: layered anisotropic infrared semiconductors with highly tunable compositions and properties. Advanced Materials 27(30), 44234429.

Long M., Gao A., Wang P., Xia H., Ott C., Pan C., Fu Y., Liu E., Chen X., Lu W., Nilges T., Xu J., Wang X., Hu W., Miao F., 2017. Room temperature high-detectivity mid-infrared photodetectors based on black arsenic phosphorus. Scientific Advances, 3(6), e1700589.

Yuan S., Shen C., Deng B., Chen X., Guo Q., Ma Y., Abbas A., Liu B., Haiges R., Ott C., Nilges T., Watanabe K., Taniguchi T., Sinai O., Naveh D., Zhou C., Xia F., 2018. Air-stable roomtemperature mid-infrared photodetectors based on hBN/black arsenic phosphorus/hBN Heterostructures. Nano Letters, 18(5), 3172-3179.

Ryzhii V., Ryzhii M., Svintsov D., Leiman V., Mitin V., Shur M.S., Otsuji T., 2017. Infrared photodetectors based on graphene van der Waals heterostructures. Infrared Physics and Technology, 84, 72-81.

Ryzhii V., Ryzhii M., Leiman V., Mitin V., Shur M. S., Otsuji T., 2017. Effect of doping on the characteristics of infrared photodetectors based on van der Waals heterostructures with multiple graphene layers. Journal of Applied Physics, 122(5), 054505.
Ryzhii V., Ryzhii M., Svintsov D., Leiman V., Mitin V., Shur M. S., Otsuji T., 2017. Nonlinear response of infrared photodetectors based on van der Waals heterostructures with graphene layers. Optics Express, 25(5), 5536.

Ryzhii V., Otsuji T., Karasik V.E., Ryzhii M., Leiman V.G., Mitin V., Shur M.S., 2018. Comparison of intersubband quantum-well and interband graphene-layer infrared photodetectors. IEEE Journal of Quantum Electronics, 54(2), 2797912.

Ryzhii V., Otsuji T., Ryzhii M., Ponomarev D.S., Karasik V.E., Leiman V.G., Mitin V., Shur M.S., 2018. Electrical modulation of terahertz radiation using graphene-phosphorene heterostructures. Semiconductor Science and Technology, 33(12), 124010 .

Ryzhii V., Otsuji T., Ryzhii M., Dubinov A.A., Aleshkin V.Ya., Karasik V.E., Shur M.S., 2019. Amplification of surface plasmons in grapheneblack phosphorus injection laser heterostructures. arXiv: 1901.00580 .

Ryzhii V., Ryzhii M., Otsuji T., Karasik V.E., Leiman V.G., Mitin V, Shur M.S., 2019. Negative terahertz conductivity at vertical carrier injection in a black-arsenic-phosphorus-graphene heterostructure integrated with a light-emitting diode. arXiv:1901.10755.

Ryzhii V., Ryzhii M., Ponomarev D.S., Leiman V. G., Mitin V., Shur M.S., Otsuji T., 2019. Negative photoconductivity and hot-carrier bolometric detection of terahertz radiation in graphenephosphorene hybrid structures. Journal of Applied Physics, 125(15), 151608. 